\title{
A dimension conjecture for $q$-analogues of multiple zeta values
}

\author{
Henrik Bachmann, Ulf Kühn
}

October 15, 2018

\begin{abstract}
We study a class of $q$-analogues of multiple zeta values given by certain formal $q$-series with rational coefficients. After introducing a notion of weight and depth for these $q$-analogues of multiple zeta values we present dimension conjectures for the spaces of their weight- and depth-graded parts, which have a similar shape as the conjectures of Zagier and Broadhurst-Kreimer for multiple zeta values.
\end{abstract}

\section{Introduction}

Multiple zeta values are real numbers appearing in various areas of mathematics and theoretical physics. By a $q$-analogue of these numbers one usually understand $q$-series, which degenerate to multiple zeta values as $q \rightarrow 1$. The algebraic structure of several models of $q$-analogues has been the subject of recent research (see [27] for an overview). Besides a conjecture of Okounkov in [17] for the dimension of the weight-graded spaces for a specific such model, no conjectures for the dimensions of the spaces of any of these $q$-analogues in a given weight and depth have occurred in the literature. The purpose of this work is to introduce a space of $q$-series which contains a lot of these models and to present conjectures on the dimensions of their weight- and depth-graded parts. For natural numbers $s_{1} \geq 2, s_{2}, \ldots, s_{l} \geq 1$ define the multiple zeta value (MZV)

$$
\zeta\left(s_{1}, \ldots, s_{l}\right)=\sum_{n_{1}>\cdots>n_{l}>0} \frac{1}{n_{1}^{s_{1}} \ldots n_{l}^{s_{l}}} .
$$

By $s_{1}+\cdots+s_{l}$ we denote its weight, by $l$ its depth and we write $\mathcal{Z}$ for the $\mathbb{Q}$-vector space spanned by all MZVs. It is a well-known fact that the space $\mathcal{Z}$ is a $\mathbb{Q}$-algebra and that there are two different ways, known as respectively the stuffle and shuffle product formulas, which express the product of two MZVs as a $\mathbb{Q}$-linear combination of MZVs. 
These two ways of writing the product gives a large family of $\mathbb{Q}$-linear relations between MZVs in a fixed weight, known as double shuffle relations. Conjecturally all relations between MZVs follow from these type of relations. In particular it is conjectured that the algebra $\mathcal{Z}$ is graded by the weight. Let $\mathcal{Z}_{k}$ denote the $\mathbb{Q}$-vector space spanned by the MZVs of weight $k$, then there is the following famous dimension conjecture due to Zagier:

Conjecture 1.1 (Zagier [25]). The following identity holds:

$$
\sum_{k \geq 0} \operatorname{dim}_{\mathbb{Q}}\left(\mathcal{Z}_{k}\right) x^{k}=\frac{1}{1-x^{2}-x^{3}} .
$$

A stronger version of this conjecture was later proposed by Hoffman ([13]), which states that the $\zeta\left(s_{1}, \ldots, s_{l}\right)$ with $s_{j} \in\{2,3\}$ form a basis of $\mathcal{Z}$. So far it is only known, due to a result of Brown ([7]), that these MZVs span the space $\mathcal{Z}$. Conjecture 1.1 has a refinement by Broadhurst and Kreimer who proposed the following conjecture on the dimension of the weight- and depth-graded parts:

Conjecture 1.2 (Broadhurst-Kreimer [6]). The generating series of the dimensions of the weight-and depth-graded parts of multiple zeta values is given by

$$
\sum_{k, l \geq 0} \operatorname{dim}_{\mathbb{Q}}\left(\operatorname{gr}_{l}^{\mathrm{D}} \mathcal{Z}_{k}\right) x^{k} y^{l}=\frac{1+\mathrm{E}_{2}(x) y}{1-\mathrm{O}_{3}(x) y+\mathrm{S}(x) y^{2}-\mathrm{S}(x) y^{4}},
$$

where

$$
\mathrm{E}_{2}(x)=\frac{x^{2}}{1-x^{2}}, \quad \mathrm{O}_{3}(x)=\frac{x^{3}}{1-x^{2}}, \quad \mathrm{~S}(x)=\frac{x^{12}}{\left(1-x^{4}\right)\left(1-x^{6}\right)}
$$

Observe that $\mathrm{E}_{2}(x)$ (resp. $\mathrm{O}_{3}(x)$ ) is the generating series of the number of even (resp. odd) zeta values and $\mathrm{S}(x)$ is the generating series for the dimensions of cusp forms for $\mathrm{SL}_{2}(\mathbb{Z})$. Furthermore, by setting $y=1$ on the right-hand side of the BroadhurstKreimer conjecture one obtains precisely the right-hand side in the Zagier conjecture. We are interested in conjectures similar to the above in the context of $q$-analogues of multiple zeta values. There are various different models of $q$-analogues for multiple zeta values. For most of these models the algebraic setup, i.e. analogues of the stuffle and the shuffle product, is well understood (See for example [3], [9], [20], [21], [27]). The problem of understanding the dimension of the weight-graded spaces has been considered in [3], 44, [9], [17], 20], 21], 27]. On the other hand possible analogues of the Broadhurst-Kreimer conjecture for these $q$-analogues have not been proposed yet. Now we will define the $q$-analogues of multiple zeta values we consider in this paper. 
For $s_{1}, \ldots, s_{l} \geq 1$ and polynomials $Q_{1}(t) \in t \mathbb{Q}[t]$ and $Q_{2}(t) \ldots, Q_{l}(t) \in \mathbb{Q}[t]$ we define

$$
\zeta_{q}\left(s_{1}, \ldots, s_{l} ; Q_{1}, \ldots, Q_{l}\right)=\sum_{n_{1}>\cdots>n_{l}>0} \frac{Q_{1}\left(q^{n_{1}}\right) \ldots Q_{l}\left(q^{n_{l}}\right)}{\left(1-q^{n_{1}}\right)^{s_{1}} \cdots\left(1-q^{n_{l}}\right)^{s_{l}}} .
$$

This series can be seen as a $q$-analogue 1 of $\zeta\left(s_{1}, \ldots, s_{l}\right)$, since we have for $s_{1}>1$

$$
\lim _{q \rightarrow 1}(1-q)^{s_{1}+\cdots+s_{l}} \zeta_{q}\left(s_{1}, \ldots, s_{l} ; Q_{1}, \ldots, Q_{l}\right)=Q_{1}(1) \ldots Q_{l}(1) \cdot \zeta\left(s_{1}, \ldots, s_{l}\right)
$$

We only consider the case where $\operatorname{deg}\left(Q_{j}\right) \leq s_{j}$ and consider the following $\mathbb{Q}$-algebra:

$$
\mathcal{Z}_{q}:=\left\langle\zeta_{q}\left(s_{1}, \ldots, s_{l} ; Q_{1}, \ldots, Q_{l}\right) \mid l \geq 0, s_{1}, \ldots, s_{l} \geq 1, \operatorname{deg}\left(Q_{j}\right) \leq s_{j}\right\rangle_{\mathbb{Q}} .
$$

Contrary to the case of MZVs, the number $s_{1}+\cdots+s_{l}$ does not give a good notion of weight for the $\zeta_{q}$, since for example $\zeta_{q}(s ; Q)=\zeta_{q}(s+1, Q \cdot(1-t))$. Also the number $l$ will not be used to define the depth. Instead we will consider a class of $q$-series which also span the space $\mathcal{Z}_{q}$ and use these series to define a weight and a depth filtration on $\mathcal{Z}_{q}$. For $s_{1}, \ldots, s_{l} \geq 1, r_{1}, \ldots, r_{l} \geq 0$ these $q$-series are given by

$$
\left[\begin{array}{l}
s_{1}, \ldots, s_{l} \\
r_{1}, \ldots, r_{l}
\end{array}\right]:=\sum_{\substack{u_{1}>\cdots>u_{l}>0 \\
v_{1}, \ldots, v_{l}>0}} \frac{u_{1}^{r_{1}}}{r_{1} !} \ldots \frac{u_{l}^{r_{l}}}{r_{l} !} \cdot \frac{v_{1}^{s_{1}-1} \ldots v_{l}^{s_{l}-1}}{\left(s_{1}-1\right) ! \ldots\left(s_{l}-1\right) !} \cdot q^{u_{1} v_{1}+\cdots+u_{l} v_{l}} \in \mathbb{Q}[[q]] .
$$

We refer to these $q$-series as bi-brackets of depth $l$ and weight $s_{1}+\cdots+s_{l}+r_{1}+\cdots+r_{l}$. They were introduced by the first author in [1] and their algebraic structure is wellunderstood and described in the papers [1], [2], [3], [30]. The bi-brackets have a natural connection to quasi-modular forms (for $\mathrm{SL}_{2}(\mathbb{Z})$ ), since for even $k$ the Fourier expansion of the classical Eisenstein series $G_{k}$ of weight $k$ is given by $\left[\begin{array}{l}k \\ 0\end{array}\right]$ plus an appropriate constant term. In particular the space of quasi-modular forms with rational coefficients, which is given by $\mathbb{Q}\left[G_{2}, G_{4}, G_{6}\right]$, is a sub-algebra of the space $\mathcal{Z}_{q}$.

As we will see in Theorem 2.3 the bi-brackets span the space $\mathcal{Z}_{q}$ and therefore we can define a weight and a depth filtration by using the notion of weight and depth of bibrackets. We point to the fact that $\mathcal{Z}_{q}$ is not graded by the weight, i.e. the weight graded spaces $\operatorname{gr}_{k}^{W} \mathcal{Z}_{q}$ are in general not isomorphic to the $\mathbb{Q}$-vector spaces spanned by bi-brackets of weight $k$. In analogy to the Zagier and Broadhurst-Kreimer conjecture we conjecture the following.

\footnotetext{
${ }^{1}$ These type of series are often called modified $q$-analogues of multiple zeta values, since one needs to multiply by $(1-q)^{s_{1}+\cdots+s_{l}}$ before taking the limit $q \rightarrow 1$
} 
Conjecture 1.3. (i) The dimension of the weight graded parts of $\mathcal{Z}_{q}$ is given by

$$
\sum_{k \geq 0} \operatorname{dim}_{\mathbb{Q}}\left(\operatorname{gr}_{k}^{W} \mathcal{Z}_{q}\right) x^{k}=\frac{1}{1-x-x^{2}-x^{3}+x^{6}+x^{7}+x^{8}+x^{9}}
$$

(ii) The dimension of the weight and depth graded parts of $\mathcal{Z}_{q}$ is given by

$$
\sum_{k, l \geq 0} \operatorname{dim}_{\mathbb{Q}}\left(\operatorname{gr}_{k, l}^{\mathrm{W}, \mathrm{D}} \mathcal{Z}_{q}\right) x^{k} y^{l}=\frac{1+\mathrm{D}(x) \mathrm{E}_{2}(x) y+\mathrm{D}(x) \mathrm{S}(x) y^{2}}{1-\mathrm{a}_{1}(x) y+\mathrm{a}_{2}(x) y^{2}-\mathrm{a}_{3}(x) y^{3}-\mathrm{a}_{4}(x) y^{4}+\mathrm{a}_{5}(x) y^{5}}
$$

where $\mathrm{D}(x)=1 /\left(1-x^{2}\right), \mathrm{O}_{1}(x)=x /\left(1-x^{2}\right)$ and $\mathrm{E}_{2}(x), \mathrm{S}(x)$ are as in Conjecture 1.2 and

$$
\begin{array}{ll}
\mathrm{a}_{1}(x)=\mathrm{D}(x) \mathrm{O}_{1}(x), & \mathrm{a}_{2}(x)=\mathrm{D}(x) \sum_{k \geq 1} \operatorname{dim}_{\mathbb{Q}}\left(M_{k}\left(\mathrm{SL}_{2}(\mathbb{Z})\right)^{2} x^{k},\right. \\
\mathrm{a}_{3}(x)=\mathrm{a}_{5}(x)=\mathrm{O}_{1}(x) \mathrm{S}(x), & \mathrm{a}_{4}(x)=\mathrm{D}(x) \sum_{k \geq 1} \operatorname{dim}_{\mathbb{Q}}\left(S_{k}\left(\mathrm{SL}_{2}(\mathbb{Z})\right)^{2} x^{k}\right.
\end{array}
$$

Here $M_{k}\left(\mathrm{SL}_{2}(\mathbb{Z})\right)$ and $S_{k}\left(\mathrm{SL}_{2}(\mathbb{Z})\right)$ denote the spaces of modular forms and cusp forms for $\mathrm{SL}_{2}(\mathbb{Z})$ of weight $k$.

Note that setting $y=1$ in (ii) implies (i), this holds because of the formula

$$
\sum_{k \geq 0} \operatorname{dim}_{\mathbb{Q}}\left(M_{k}\left(\mathrm{SL}_{2}(\mathbb{Z})\right)^{2} x^{k}=\frac{1+x^{12}}{\left(1-x^{4}\right)\left(1-x^{6}\right)\left(1-x^{12}\right)}\right.
$$

which is straightforward to prove.

In the Broadhurst-Kreimer conjecture the numerator $1+\mathrm{E}_{2}(x) y$ can be interpreted as the generating series of $\operatorname{dim}_{\mathbb{Q}} \operatorname{gr}_{k, l}^{W, D} \mathbb{Q}[\zeta(2)]$, i.e.

$$
\sum_{k, l \geq 0} \operatorname{dim}_{\mathbb{Q}} \operatorname{gr}_{k, l}^{\mathrm{W}, \mathrm{D}}(\mathbb{Q}[\zeta(2)]) x^{k} y^{l}=1+\mathrm{E}_{2}(x) y
$$

As we will see in Proposition 2.5 the numerator in Conjecture 1.3 (ii) is essentially the generating series for the weight- and depth-graded dimensions of the quasi-modular forms, since $\mathrm{D}(x) \mathrm{E}_{2}(x)$ counts the number of Eisenstein series and their derivatives and $\mathrm{D}(x) \mathrm{S}(x)$ corresponds to the number of cusp forms and their derivatives. Therefore it is reasonable to expect that

$$
\sum_{k, l \geq 0} \operatorname{dim}_{\mathbb{Q}} \operatorname{gr}_{k, l}^{\mathrm{W}, \mathrm{D}}\left(\mathbb{Q}\left[G_{2}, G_{4}, G_{6}\right]\right) x^{k} y^{l} \stackrel{?}{=} 1+\mathrm{D}(x) \mathrm{E}_{2}(x) y+\mathrm{D}(x) \mathrm{S}(x) y^{2} .
$$


In some sense the quasi-modular forms in the context of $q$-analogues of multiple zeta values play the role of the even single zeta values (see also [11], [28]).

For $k \leq 15$ we determined, by calculating a large number of coefficients, lower bounds for $\operatorname{dim}_{\mathbb{Q}}\left(\operatorname{gr}_{k}^{W} \mathcal{Z}_{q}\right)$, which equal the expected dimensions in Conjecture 1.3 (i). Furthermore, see Theorem 3.3 below, Conjecture 1.3 (i) actually holds for $k \leq 7$. For the refined Conjecture 1.3 (ii) our computer experiments provide us with lower bounds, which again equal the expected dimensions, in the range given by Table 4 on page 17.

\section{Acknowledgments}

We would like to thank N. Matthes for the careful reading of our manuscript and his valuable comments. The first author would also like to thank the Max-Planck Institute for Mathematics in Bonn for hospitality and support.

\section{$2 q$-analogues of MZVs and bi-brackets}

Usually a function $f(q)$ is called a $q$-analogue of multiple zeta value, if $\lim _{q \rightarrow 1} f(q)$ is a multiple zeta value. There are various different models of $q$-analogues in the literature (See [27] for a nice overview). One of the first models was studied by Bradley [5] and Zhao [26] independently. This model is given for $s_{1} \geq 2, s_{2}, \ldots s_{l} \geq 1$ by the $q$-series

$$
\sum_{n_{1}>\cdots>n_{l}>0} \frac{q^{\left(s_{1}-1\right) n_{1}} \cdots q^{\left(s_{l}-1\right) n_{l}}}{\left\{n_{1}\right\}_{q}^{s_{1}} \cdots\left\{n_{l}\right\}_{q}^{s_{l}}}
$$

with $\{n\}_{q}=\frac{1-q^{n}}{1-q}$ being the usual $q$-integer. Taking the limit $q \rightarrow 1$ in above sum one obtains $\zeta\left(s_{1}, \ldots, s_{l}\right)$. For a cleaner description of the algebraic structure and (in our case) a connection to modular forms it is convenient to consider a modified version of (2.1) by removing the factor $(1-q)^{s_{1}+\cdots+s_{l}}$, i.e. to consider the series

$$
\zeta_{q}^{\mathrm{BZ}}\left(s_{1}, \ldots, s_{l}\right)=\sum_{n_{1}>\cdots>n_{l}>0} \frac{q^{\left(s_{1}-1\right) n_{1}} \ldots q^{\left(s_{l}-1\right) n_{l}}}{\left(1-q^{n_{1}}\right)^{s_{1}} \cdots\left(1-q^{n_{l}}\right)^{s_{l}}},
$$

which then satisfies $\lim _{q \rightarrow 1}(1-q)^{s_{1}+\cdots+s_{l}} \zeta_{q}\left(s_{1}, \ldots, s_{l}\right)=\zeta\left(s_{1}, \ldots, s_{l}\right)$.

In a greater generality we will consider for $s_{1}, \ldots, s_{l} \geq 1$ and polynomials $Q_{1}(t) \in t \mathbb{Q}[t]$ and $Q_{2}(t) \ldots, Q_{l}(t) \in \mathbb{Q}[t]$ sums of the form

$$
\zeta_{q}\left(s_{1}, \ldots, s_{l} ; Q_{1}, \ldots, Q_{l}\right)=\sum_{n_{1}>\cdots>n_{l}>0} \frac{Q_{1}\left(q^{n_{1}}\right) \ldots Q_{l}\left(q^{n_{l}}\right)}{\left(1-q^{n_{1}}\right)^{s_{1}} \ldots\left(1-q^{n_{l}}\right)^{s_{l}}} .
$$

The condition $Q_{1}(t) \in t \mathbb{Q}[t]$ ensures that this is an element in $\mathbb{Q}[[q]]$. In contrast to 
(2.2) we also allow $s_{1}=1$ in our setup, i.e. we also include $q$-analogues of the nonconvergent multiple zeta values. In the case $s_{1}>1$ we can (by the same arguments as in [4] Proposition 6.4) again take the limit $q \rightarrow 1$ after multiplying by $(1-q)^{s_{1}+\cdots+s_{l}}$, which gives

$$
\lim _{q \rightarrow 1}(1-q)^{s_{1}+\cdots+s_{l}} \zeta_{q}\left(s_{1}, \ldots, s_{l} ; Q_{1}, \ldots, Q_{l}\right)=Q_{1}(1) \ldots Q_{l}(1) \cdot \zeta\left(s_{1}, \ldots, s_{l}\right)
$$

Almost all models of $q$-analogues in the literature are given by sums of the form (2.3) . In the following we always set $\zeta_{q}\left(s_{1}, \ldots, s_{l} ; Q_{1}, \ldots, Q_{l}\right)=1$ for the case $l=0$. We will consider the following spaces spanned by the series (2.3) of a particular kind

$$
\mathcal{Z}_{q}=\left\langle\zeta_{q}\left(s_{1}, \ldots, s_{l} ; Q_{1}, \ldots, Q_{l}\right) \mid l \geq 0, s_{1}, \ldots, s_{l} \geq 1, \operatorname{deg}\left(Q_{j}\right) \leq s_{j}\right\rangle_{\mathbb{Q}},
$$

where as before we always assume $Q_{1}(t) \in t \mathbb{Q}[t]$ and $Q_{2}(t) \ldots, Q_{l}(t) \in \mathbb{Q}[t]$. As we will see below $\mathcal{Z}_{q}$ is the space in which we are interested the most. For $d \geq 0$ we define the subspace $\mathcal{Z}_{q, d}=\left\langle\zeta_{q}\left(s_{1}, \ldots, s_{l} ; Q_{1}, \ldots, Q_{l}\right) \in \mathcal{Z}_{q} \mid \operatorname{deg}\left(Q_{j}\right) \leq s_{j}-d\right\rangle_{\mathbb{Q}}$. So in particular it is $\mathcal{Z}_{q}=\mathcal{Z}_{q, 0}$ and $\mathcal{Z}_{q, d+1} \subset \mathcal{Z}_{q, d}$. We also restrict to the case in which all polynomials $Q_{j}$ (not just $Q_{1}$ ) have no constant terms and therefore are elements in $t \mathbb{Q}[t]$. The resulting space is denoted by

$$
\mathcal{Z}_{q}^{\circ}=\left\langle\zeta_{q}\left(s_{1}, \ldots, s_{l} ; Q_{1}, \ldots, Q_{l}\right) \in \mathcal{Z}_{q} \mid Q_{1}, \ldots, Q_{l} \in t \mathbb{Q}[t]\right\rangle_{\mathbb{Q}}
$$

For the spaces $\mathcal{Z}_{q, d}^{\circ}$ given by $\mathcal{Z}_{q}^{\circ} \cap \mathcal{Z}_{q, d}$ it holds $\mathcal{Z}_{q}^{\circ}=\mathcal{Z}_{q, 0}^{\circ}$ and $\mathcal{Z}_{q, d+1}^{\circ} \subset \mathcal{Z}_{q, d}^{\circ}$. Notice that all of these spaces are closed under multiplication. In depth one for example it is

$$
\zeta_{q}\left(s_{1} ; Q_{1}\right) \cdot \zeta_{q}\left(s_{2} ; Q_{2}\right)=\zeta_{q}\left(s_{1}, s_{2} ; Q_{1}, Q_{2}\right)+\zeta_{q}\left(s_{2}, s_{1} ; Q_{2}, Q_{1}\right)+\zeta_{q}\left(s_{1}+s_{2} ; Q_{1} \cdot Q_{2}\right)
$$

and clearly $\operatorname{deg} Q_{1} \cdot Q_{2} \leq s_{1}+s_{2}-d$ if $\operatorname{deg} Q_{j} \leq s_{j}-d$ for $j=1,2$.

In [27] Zhao considers for $s_{1}, \ldots, s_{l}, d_{1}, \ldots, d_{l} \in \mathbb{Z}$ the series

$$
\mathfrak{z}_{q}^{d_{1}, \ldots, d_{l}}\left(s_{1}, \ldots, s_{l}\right)=\sum_{n_{1}>\cdots>n_{l}>0} \frac{q^{n_{1} d_{1}} \ldots q^{n_{l} d_{l}}}{\left(1-q^{n_{1}}\right)^{s_{1}} \cdots\left(1-q^{n_{l}}\right)^{s_{l}}}
$$

which gives an even more general setup than our $\zeta_{q}$. Especially these series can be seen as natural generators of the spaces $\mathcal{Z}_{q, d}$ and $\mathcal{Z}_{q, d}^{\circ}$ by choosing the appropriate conditions on the $d_{j}$. We will now give a short overview of different $q$-analogues of multiple zeta values, which can be written in terms of the $\zeta_{q}$ and relate them to the spaces $\mathcal{Z}_{q, d}$ and $\mathcal{Z}_{q, d}^{\circ}$.

(i) The space spanned by the Bradley-Zhao model $\zeta_{q}^{\mathrm{BZ}}=\zeta_{q}\left(s_{1}, \ldots, s_{l} ; t^{s_{1}-1}, \ldots, t^{s_{j}-1}\right)$, 
defined in (2.2), is given by 2

$$
\mathcal{Z}_{q, 1}=\left\langle\zeta_{q}^{\mathrm{BZ}}\left(s_{1}, \ldots, s_{l}\right) \mid l \geq 0, s_{1} \geq 2, s_{2}, \ldots, s_{l} \geq 1\right\rangle_{Q}
$$

(ii) Another interesting case is the Schlesinger-Zudilin model. These q-analogues are for $s_{1} \geq 1, s_{2}, \ldots, s_{l} \geq 0$ defined by

$$
\begin{aligned}
\zeta_{q}^{\mathrm{SZ}}\left(s_{1}, \ldots, s_{l}\right) & =\sum_{n_{1}>\cdots>n_{l}>0} \frac{q^{n_{1} s_{1}} \ldots q^{n_{l} s_{l}}}{\left(1-q^{n_{1}}\right)^{s_{1}} \cdots\left(1-q^{n_{l}}\right)^{s_{l}}} \\
& =\zeta_{q}\left(s_{1}, \ldots, s_{l} ; t^{s_{1}}, \ldots, t^{s_{j}}\right) .
\end{aligned}
$$

The space spanned by these series is, using the same argument as in (i), given by

$$
\mathcal{Z}_{q}=\left\langle\zeta_{q}^{\mathrm{SZ}}\left(s_{1}, \ldots, s_{l}\right) \mid l \geq 0, s_{1} \geq 1, s_{2}, \ldots, s_{l} \geq 0\right\rangle_{\mathbb{Q}}
$$

Originally defined by Schlesinger [18] and Zudilin [29] for the cases $s_{1} \geq 2, s_{2}, \ldots, s_{l} \geq$ 1 , it was observed in [20] and further discussed in [9] that the algebraic setup, especially the shuffle product analogue, for this model can be described nicely by allowing $s_{1} \geq 1, s_{2}, \ldots, s_{l} \geq 0$. Restricting to $s_{1}, \ldots, s_{l} \geq 1$ we get the subspace

$$
\mathcal{Z}_{q}^{\circ}=\left\langle\zeta_{q}^{\mathrm{SZ}}\left(s_{1}, \ldots, s_{l}\right) \mid l \geq 0, s_{1}, \ldots, s_{l} \geq 1\right\rangle_{\mathbb{Q}}
$$

(iii) In [22] Ohno-Okuda-Zudilin define for $s_{1}, \ldots, s_{l} \in \mathbb{Z}$ the series

$$
\zeta_{q}^{\mathrm{OOZ}}\left(s_{1}, \ldots, s_{l}\right)=\sum_{n_{1}>\cdots>n_{l}>0} \frac{q^{n_{1}}}{\left(1-q^{n_{1}}\right)^{s_{1}} \cdots\left(1-q^{n_{l}}\right)^{s_{l}}} .
$$

In the case $s_{1}, \ldots, s_{l} \geq 1$ these can be written as $\zeta_{q}\left(s_{1}, \ldots, s_{l} ; t, 1, \ldots, 1\right) \in \mathcal{Z}_{q}$, but the space spanned by (2.6) for $s_{1}, \ldots, s_{j} \geq 1$ is a priori not given by one of the $\mathcal{Z}_{q, d}$ or $\mathcal{Z}_{q, d}^{\circ}$.

(iv) For $s_{1}, \ldots, s_{l} \geq 2$ Okounkov chooses the following polynomials in [17]

$$
Q_{j}^{O}(t)= \begin{cases}t^{\frac{s_{j}}{2}} & s_{j}=2,4,6, \ldots \\ t^{\frac{s_{j}-1}{2}}(1+t) & s_{j}=3,5,7, \ldots\end{cases}
$$

and defines $\mathrm{Z}\left(s_{1}, \ldots, s_{l}\right)=\zeta_{q}\left(s_{1}, \ldots, s_{l} ; Q_{1}^{O}, \ldots, Q_{l}^{O}\right)$. With the same arguments as before (see also the proof of Theorem 2.3 (iii)) the span of these series is given

${ }^{2}$ This follows easily from the fact that $t^{j-1}(1-t)^{s-j}$ with $j=1, \ldots, s$ (resp. $\left.j=2, \ldots, s\right)$ forms a basis of $\{Q \in \mathbb{Q}[t] \mid \operatorname{deg} Q \leq s-1\}$ (resp. $\{Q \in t \mathbb{Q}[t] \mid \operatorname{deg} Q \leq s-1\}$ ). 
by

$$
\mathcal{Z}_{q, 1}^{\circ}=\left\langle\mathrm{Z}\left(s_{1}, \ldots, s_{l}\right) \mid l \geq 0, s_{1}, \ldots, s_{l} \geq 2\right\rangle_{\mathbb{Q}} .
$$

Although the space $\mathcal{Z}_{q}$ seems to be much larger than the space $\mathcal{Z}_{q}^{\circ}$, we expect that they both coincide (Conjecture 3.2 (B2) below) and therefore every $\zeta_{q}^{\text {SZ }}$ should be expressible as a linear combination of $\zeta_{q}^{\mathrm{SZ}}\left(s_{1}, \ldots, s_{l}\right)$ with $s_{1}, \ldots, s_{l} \geq 1$. In [9] (Theorem 5.5) such an expression for $\zeta_{q}^{\mathrm{OOZ}}$ in terms of $\zeta_{q}^{\mathrm{SZ}}$ is given, which in turn can be seen as a special case of that conjecture.

Remark 2.1. As seen in the example above, the polynomials $Q_{j}$ often depend just on $s_{j}$. For these types of q-analogues one can also define subspaces of $\mathcal{Z}_{q}$ in the following way: Suppose that $\left\{Q_{s}\right\}_{s \geq 1}$ is a family of polynomials, where for all $s_{1}, s_{2} \geq 1$ there exists numbers $\lambda_{j}^{s_{1}, s_{2}} \in \mathbb{Q}$ with $j \geq 1$ and $\lambda_{j}^{s_{1}, s_{2}}=0$ for almost all $j$, such that

$$
Q_{s_{1}}(t) \cdot Q_{s_{2}}(t)=\sum_{j=1}^{\infty} \lambda_{j}^{s_{1}, s_{2}} Q_{j}(t)(1-t)^{s_{1}+s_{j}-j}
$$

Then the space spanned by all $\zeta_{q}\left(s_{1}, \ldots, s_{l} ; Q_{s_{1}}, \ldots, Q_{s_{l}}\right)$ is a sub-algebra of $\mathcal{Z}_{q}$. This also gives an example of a so called quasi-shuffle algebra as described in [14]. For this one can define for $a, b \geq 1$ the product $z_{a} \diamond z_{b}=\sum_{j=1}^{\infty} \lambda_{j}^{a, b} z_{j}$ with the same notation as used in the first section of [14]. This was for example done in [4] for the space $\mathcal{Z}_{q}^{\circ}$.

\subsection{Bi-brackets as $q$-analogues of MZVs}

In this section we will consider the $q$-series from the introduction in more detail and explain their connection to $q$-analogues of multiple zeta values in the section before.

Definition 2.2. (i) For $s_{1}, \ldots, s_{l} \geq 1, r_{1}, \ldots, r_{l} \geq 0$ we define the following q-series

$$
\left[\begin{array}{l}
s_{1}, \ldots, s_{l} \\
r_{1}, \ldots, r_{l}
\end{array}\right]:=\sum_{\substack{u_{1}>\ldots>u_{l}>0 \\
v_{1}, \ldots, v_{l}>0}} \frac{u_{1}^{r_{1}}}{r_{1} !} \ldots \frac{u_{l}^{r_{l}}}{r_{l} !} \cdot \frac{v_{1}^{s_{1}-1} \ldots v_{l}^{s_{l}-1}}{\left(s_{1}-1\right) ! \ldots\left(s_{l}-1\right) !} \cdot q^{u_{1} v_{1}+\cdots+u_{l} v_{l}} \in \mathbb{Q}[[q]] .
$$

We refer to these $q$-series as bi-brackets of depth $l$ and of weight $s_{1}+\cdots+s_{l}+$ $r_{1}+\cdots+r_{l}$.

(ii) For $r_{1}=\cdots=r_{l}=0$ we write

$$
\left[s_{1}, \ldots, s_{l}\right]:=\left[\begin{array}{c}
s_{1}, \ldots, s_{l} \\
0, \ldots, 0
\end{array}\right] .
$$

The series $\left[s_{1}, \ldots, s_{l}\right]$, which we call brackets, were introduced and studied in $[4]$. 
The bi-brackets also have an alternative form, which we will use now. For this recall that the Eulerian polynomials (See for example [10]) are defined by

$$
\frac{t P_{s-1}(t)}{(1-t)^{s}}=\sum_{d=1}^{\infty} d^{s-1} t^{d}
$$

For $s>1$ the polynomials $t P_{s-1}(t)$ have degree $s-1$ and in the case $s=1$ it is $t P_{0}(t)=t$. By definition of the bi-brackets it is then clear that

$$
\left[\begin{array}{l}
s_{1}, \ldots, s_{l} \\
r_{1}, \ldots, r_{l}
\end{array}\right]=\sum_{n_{1}>\cdots>n_{l}>0} \prod_{j=1}^{l}\left(\frac{n_{j}^{r_{j}}}{r_{j} !} \cdot \frac{q^{n_{j}} P_{s_{j}-1}\left(q^{n_{j}}\right)}{\left(s_{j}-1\right) ! \cdot\left(1-q^{n_{j}}\right)^{s_{j}}}\right)
$$

We will now see that the spaces spanned 3 by the bi-brackets and brackets are exactly given by the spaces $\mathcal{Z}_{q}$ and $\mathcal{Z}_{q}^{\circ}$ respectively.

Theorem 2.3. The following equalities hold

$$
\begin{gathered}
\mathcal{Z}_{q}=\left\langle\left[\begin{array}{l}
s_{1}, \ldots, s_{l} \\
r_{1}, \ldots, r_{l}
\end{array}\right] \mid l \geq 0, s_{1}, \ldots, s_{l} \geq 1, r_{1}, \ldots, r_{l} \geq 0\right\rangle_{\mathbb{Q}} . \\
\mathcal{Z}_{q}^{\circ}=\left\langle\left[s_{1}, \ldots, s_{l}\right] \mid l \geq 0, s_{1}, \ldots, s_{l} \geq 1\right\rangle_{Q} . \\
\mathcal{Z}_{q, 1}^{\circ}=\left\langle\left[s_{1}, \ldots, s_{l}\right] \mid l \geq 0, s_{1}, \ldots, s_{l} \geq 2\right\rangle_{Q} .
\end{gathered}
$$

Proof. Since for all $s \geq 1$ it is $P_{s-1}(1) \neq 0$ the polynomials $t P_{j-1}(t)(1-t)^{s-j}$ with $j=1, \ldots, s$ form a basis of the space $\{Q \in t \mathbb{Q}[t] \mid \operatorname{deg} Q \leq s\}$. In particular for every polynomial $Q$ in this space there exists coefficients $\alpha_{j} \in \mathbb{Q}$ with

$$
\frac{Q(t)}{(1-t)^{s}}=\sum_{j=1}^{s} \alpha_{j} \frac{t P_{j-1}(t)}{(1-t)^{j}}
$$

from which the statement (ii) follows. Also (iii) follows, since for $d=1$ the condition $\mathbb{Q}_{j}(t) \in t \mathbb{Q}[t]$ and $\operatorname{deg} Q_{j} \leq s_{j}-1$ implies $s_{j} \geq 2$ for all $j=1, \ldots, l$. One can also see that

$$
\left\langle\left[s_{1}, \ldots, s_{l}\right] \mid l \geq 0, s_{1}, \ldots, s_{l} \geq 2\right\rangle_{Q}=\left\langle\zeta_{q}^{\mathrm{BZ}}\left(s_{1}, \ldots, s_{l}\right) \mid l \geq 0, s_{1}, \ldots, s_{l} \geq 2\right\rangle_{Q} .
$$

To prove (i) we will first show the inclusion ' $\subseteq$ ', i.e. that every $\zeta_{q}\left(s_{1}, \ldots, s_{l} ; Q_{1}, \ldots, Q_{l}\right)$ can be written in terms of bi-brackets. For this we need to see what happens if one of

\footnotetext{
${ }^{3}$ In the articles [1], [2] and [4] these spaces were denoted $\mathcal{B D}$ and $\mathcal{M D}$.
} 
the $Q_{2}, \ldots, Q_{l}$ has a constant term. Without loss of generality we can, by the proof of (ii), focus on the cases $Q_{i}(t)=1$ for a $2 \leq i \leq l$. Since for all $s \geq 1$ it is

$$
\frac{1}{(1-t)^{s}}=1+\sum_{m=1}^{s} \frac{t}{(1-t)^{m}}
$$

we can write

$$
\sum_{n_{1}>\cdots>n_{l}>0} \prod_{j=1}^{l} \frac{Q_{j}\left(q^{n_{j}}\right)}{\left(1-q^{n_{j}}\right)^{s_{j}}}=\sum_{n_{1}>\cdots>n_{l}>0} \prod_{\substack{j=1 \\ j \neq i}}^{l} \frac{Q_{j}\left(q^{n_{j}}\right)}{\left(1-q^{n_{j}}\right)^{s_{j}}}+\sum_{\substack{n_{1}>\cdots>n_{l}>0 \\ 1 \leq m \leq s_{i}}} \frac{q^{n_{i}}}{\left(1-q^{n_{i}}\right)^{m}} \prod_{\substack{j=1 \\ j \neq i}}^{l} \frac{Q_{j}\left(q^{n_{j}}\right)}{\left(1-q^{n_{j}}\right)^{s_{j}}} .
$$

For the the second sum on the right-hand side we can again use (2.8). For the first sum we obtain (by setting $n_{l+1}=0$ )

$$
\sum_{n_{1}>\cdots>n_{l}>0} \prod_{\substack{j=1 \\ j \neq i}}^{l} \frac{Q_{j}\left(q^{n_{j}}\right)}{\left(1-q^{n_{j}}\right)^{s_{j}}}=\sum_{n_{1}>\cdots>n_{i-1}>n_{i+1}>\cdots>n_{l}>0}\left(n_{i-1}-n_{i+1}-1\right) \prod_{\substack{j=1 \\ j \neq i}}^{l} \frac{Q_{j}\left(q^{n_{j}}\right)}{\left(1-q^{n_{j}}\right)^{s_{j}}}
$$

Repeating this for all $2 \leq i \leq l$ with $Q_{i}(t)=1$ we obtain sums of the form (2.7) from which we deduce ' $\subseteq$ '.

Now to prove ' $\supseteq$ ' we first define for $m \geq 0$ the polynomials $p_{m}(n)$ by $p_{0}(n)=1$ and

$$
p_{m}(n)=\sum_{n>N_{1}>\cdots>N_{m}>0} 1
$$

The $p_{m}(n)$ is a polynomial in $n$ of degree $m$ and therefore we can always find $c_{m}(r) \in \mathbb{Q}$ with $n^{r}=\sum_{m=0}^{r} c_{m}(r) p_{m}(n)$. The idea is now to replace $n_{j}^{r_{j}}$ in the definition of the bibrackets by $\sum_{m_{j}=0}^{r_{j}} c_{m_{j}}\left(r_{j}\right) p_{m_{j}}\left(n_{j}\right)$ and then use (2.9) to get sums which can be written in terms of the $\zeta_{q}$. We illustrate this in the depth two case from which the general case becomes clear. We have with $\kappa=\left(s_{1}-1\right) !\left(s_{2}-1\right) ! r_{1} ! r_{2}$ !

$$
\begin{aligned}
& \kappa \cdot\left[\begin{array}{l}
s_{1}, s_{2} \\
r_{1}, r_{2}
\end{array}\right]=\sum_{n_{1}>n_{2}>0} \frac{n_{1}^{r_{1}} q^{n_{1}} P_{s_{1}-1}\left(q^{n_{1}}\right)}{\left(1-q^{n_{1}}\right)^{s_{1}}} \frac{n_{2}^{r_{2}} q^{n_{2}} P_{s_{2}-1}\left(q^{n_{2}}\right)}{\left(1-q^{n_{2}}\right)^{s_{2}}} \\
& =\sum_{0 \leq m_{2} \leq r_{2}} c_{m_{2}}\left(r_{2}\right) \sum_{n_{1}>n_{2}>N_{1}>\cdots>N_{m_{2}}>0} \frac{n_{1}^{r_{1}} q^{n_{1}} P_{s_{1}-1}\left(q^{n_{1}}\right)}{\left(1-q^{n_{1}}\right)^{s_{1}}} \frac{q^{n_{2}} P_{s_{2}-1}\left(q^{n_{2}}\right)}{\left(1-q^{n_{2}}\right)^{s_{2}}} \\
& =\sum_{\substack{0 \leq m_{1} \leq r_{1} \\
0 \leq m_{2} \leq r_{2}}} c_{m_{1}}\left(r_{1}\right) c_{m_{2}}\left(r_{2}\right) \sum_{\substack{n_{1}>n_{2}>N_{1}>\cdots>N_{m_{2}}>0 \\
n_{1}>N_{1}^{\prime}>\cdots>N_{m_{1}}^{\prime}>0}} \frac{q^{n_{1}} P_{s_{1}-1}\left(q^{n_{1}}\right)}{\left(1-q^{n_{1}}\right)^{s_{1}}} \frac{q^{n_{2}} P_{s_{2}-1}\left(q^{n_{2}}\right)}{\left(1-q^{n_{2}}\right)^{s_{2}}} .
\end{aligned}
$$

Now considering all the possible shuffles, and possible equalities of the $N$ and the $N^{\prime}$ 
it is clear that this sum can be written as a linear combination of $\zeta_{q}$ by interpreting appearing 1 as $\left(1-q^{N}\right)\left(1-q^{N}\right)^{-1}$. For general depth $l$ the idea is the same and therefore we obtain ' $\supseteq$ ' from which (i) follows.

As an example how to write a bi-bracket in terms of $\zeta_{q}$ we give the following.

$$
\begin{aligned}
{\left[\begin{array}{l}
1,1 \\
0,1
\end{array}\right] } & =\sum_{n_{1}>n_{2}>0} \frac{q^{n_{1}}}{\left(1-q^{n_{1}}\right)} \frac{n_{2} q^{n_{2}}}{\left(1-q^{n_{2}}\right)} \\
& =\sum_{n_{1}>n_{2}>0} \frac{q^{n_{1}}}{\left(1-q^{n_{1}}\right)} \frac{q^{n_{2}}}{\left(1-q^{n_{2}}\right)}+\sum_{n_{1}>n_{2}>n_{3}>0} \frac{q^{n_{1}}}{\left(1-q^{n_{1}}\right)} \frac{q^{n_{2}}}{\left(1-q^{n_{2}}\right)} \frac{1-q^{n_{3}}}{\left(1-q^{n_{3}}\right)} \\
& =\zeta_{q}(1,1 ; t, t)+\zeta_{q}(1,1,1 ; t, t, 1-t) .
\end{aligned}
$$

\subsection{Bi-brackets and quasi-modular forms}

We now define the weight and the depth filtration for the space $\mathcal{Z}_{q}$ by writing for a subset $A \subseteq \mathcal{Z}_{q}$

$$
\begin{aligned}
\operatorname{Fil}_{k}^{\mathrm{W}}(A) & :=\left\langle\left[\begin{array}{l}
s_{1}, \ldots, s_{l} \\
r_{1}, \ldots, r_{l}
\end{array}\right] \in A \mid 0 \leq l \leq k, s_{1}+\cdots+s_{l}+r_{1}+\cdots+r_{l} \leq k\right\rangle_{\mathbb{Q}} \\
\operatorname{Fil}_{l}^{\mathrm{D}}(A) & :=\left\langle\left[\begin{array}{l}
s_{1}, \ldots, s_{t} \\
r_{1}, \ldots, r_{t}
\end{array}\right] \in A \mid t \leq l\right\rangle_{\mathbb{Q}} .
\end{aligned}
$$

If we consider the depth and weight filtration at the same time we use the short notation $\mathrm{Fil}_{k, l}^{\mathrm{W}, \mathrm{D}}:=\mathrm{Fil}_{k}^{\mathrm{W}} \mathrm{Fil}_{l}^{\mathrm{D}}$ and similar for the other filtrations. The associated graded spaces will be denoted by $\mathrm{gr}_{k}^{\mathrm{W}}$ and $\mathrm{gr}_{k, l}^{\mathrm{W}, \mathrm{D}}$.

Remark 2.4. (i) We point to the fact that the filtration by depth coming from bibrackets is different from the naive notion of depth for the $\zeta_{q}\left(s_{1}, \ldots, s_{l}\right)$, given as the number of variables $s_{i}$. For example, as indicated by (2.10), the $\zeta_{q}(1,1,1 ; t, t, 1-$ t) is an element in $\mathrm{Fil}_{2}^{\mathrm{D}}\left(\mathcal{Z}_{q}\right)$.

(ii) As seen before the Schlesinger-Zudilin model $\zeta_{q}^{S Z}\left(s_{1}, \ldots, s_{l}\right)$, defined in (2.5) for $s_{1} \geq 1, s_{2}, \ldots, s_{l} \geq 0$, span the space $\mathcal{Z}_{q}$ and therefore we also obtain a depth and weight filtration for these series. By the proof of Theorem 2.3 we see that $\zeta_{q}^{S Z}\left(s_{1}, \ldots, s_{l}\right) \in \mathrm{Fil}_{K, L}^{\mathrm{W}, \mathrm{D}}\left(\mathcal{Z}_{q}\right)$ with $K=s_{1}+\cdots+s_{l}+z$ and $L=l+z$, where $z=\#\left\{j \mid s_{j}=0\right\}$ is the number of $s_{j}$ which are zero.

For several reasons one should consider these filtrations to be the natural ones. First of all the multiplication in $\mathcal{Z}_{q}$ respects the depth as well as the weight grading. Secondly, on $\mathcal{Z}_{q}$ we have the derivation given by $q \frac{d}{d q}$, it increases the weight by 2 and keeps the 
depth, since we obtain directly from the definition that

$$
q \frac{d}{d q}\left[\begin{array}{l}
s_{1}, \ldots, s_{l} \\
r_{1}, \ldots, r_{l}
\end{array}\right]=\sum_{j=1}^{l}\left(s_{j}\left(r_{j}+1\right)\left[\begin{array}{l}
s_{1}, \ldots, s_{j-1}, s_{j}+1, s_{j+1}, \ldots, s_{l} \\
r_{1}, \ldots, r_{j-1}, r_{j}+1, r_{j+1}, \ldots, r_{l}
\end{array}\right]\right) .
$$

Thirdly, the classical Eisenstein series are contained in $\mathcal{Z}_{q}^{\circ} \subset \mathcal{Z}_{q}$. For example we have

$$
G_{2}=-\frac{1}{24}+[2], \quad G_{4}=\frac{1}{1440}+[4], \quad G_{6}=-\frac{1}{60480}+[6]
$$

since in depth one it is for $k>0$

$$
[k]=\sum_{\substack{u>0 \\ v>0}} \frac{v^{k-1}}{(k-1) !} q^{u v}=\frac{1}{(k-1) !} \sum_{n>0} \sum_{d \mid n} d^{k-1} q^{n}=\frac{1}{(k-1) !} \sum_{n>0} \sigma_{k-1}(n) q^{n} .
$$

The space of quasi-modular forms for $\mathrm{SL}_{2}(\mathbb{Z})$ with rational coefficients is given by $\widetilde{M}\left(\mathrm{SL}_{2}(\mathbb{Z})\right)_{\mathbb{Q}}=\mathbb{Q}\left[G_{2}, G_{4}, G_{6}\right]$ (see[16]) and therefore it is a sub-algebra of $\mathcal{Z}_{q}^{\circ}$ and $\mathcal{Z}_{q}$. It is graded by the weight, in the classical sense, and obviously $\widetilde{M}_{k}\left(\mathrm{SL}_{2}(\mathbb{Z})\right)_{\mathbb{Q}} \subset \mathrm{Fil}_{k}^{\mathrm{W}}\left(\mathcal{Z}_{q}\right)$. The derivation $q \frac{d}{d q}$ increases the weight by 2 , i.e.

$$
q \frac{d}{d q}: \widetilde{M}_{k}\left(\mathrm{SL}_{2}(\mathbb{Z})\right)_{\mathbb{Q}} \rightarrow \widetilde{M}_{k+2}\left(\mathrm{SL}_{2}(\mathbb{Z})\right)_{\mathbb{Q}}
$$

The space of quasi-modular forms has the decomposition

$$
\widetilde{M}_{k}\left(\mathrm{SL}_{2}(\mathbb{Z})\right)_{\mathbb{Q}}=\left\langle G_{k}, q \frac{d}{d q} G_{k-2}, \ldots,\left(q \frac{d}{d q}\right)^{k / 2-1} G_{2}\right\rangle_{\mathbb{Q}} \oplus \bigoplus_{i=0}^{k / 2}\left(q \frac{d}{d q}\right)^{i} S_{k-2 i}\left(\mathrm{SL}_{2}(\mathbb{Z})\right)_{\mathbb{Q}}
$$

Proposition 2.5. Set $\widetilde{\mathrm{M}}(x, t)=1+\mathrm{D}(x) \mathrm{E}_{2}(x) t+\mathrm{D}(x) \mathrm{S}(x) t^{2}$, then the generating series for the weight- and depth-graded dimensions of $\widetilde{M}\left(\mathrm{SL}_{2}(\mathbb{Z})\right)_{\mathbb{Q}} \subset \mathcal{Z}_{q}$ satisfies the coefficient-wise inequality

$$
\sum_{k, l} \operatorname{dim}_{\mathbb{Q}} \operatorname{gr}_{k, l}^{\mathrm{W}, \mathrm{D}}\left(\widetilde{M}\left(\mathrm{SL}_{2}(\mathbb{Z})\right)_{\mathbb{Q}}\right) x^{k} t^{l} \leq \widetilde{\mathrm{M}}(x, t) .
$$

Proof. The Eisenstein series and their derivatives are in the depth one subspaces. For the space of cusp forms of weight $k$ we have, by using a theorem of Zagier,

$$
S_{k}\left(\mathrm{SL}_{2}(\mathbb{Z})\right)_{\mathbb{Q}} \subset\left\langle G_{k-a} G_{a} \mid a=0, \ldots, k / 2\right\rangle_{\mathbb{Q}} \subset \mathrm{Fil}_{k, 2}^{\mathrm{W}, \mathrm{D}}\left(\mathcal{Z}_{q}\right)
$$


Finally since $q \frac{d}{d q}$ does not alter the depth we get the claim by the decomposition (2.11).

The expected equality in Proposition 2.5 would hold if the brackets [2], [4], [6] and the odd brackets [1], [3], .. together with all of their derivatives were algebraically independent, but by now only partial results for linear independence are available ([24],[28]).

Conjecture 2.6. We have a decomposition of $\mathbb{Q}$-algebras

$$
\mathcal{Z}_{q} \cong \widetilde{M}_{\mathbb{Q}}\left(\mathrm{SL}_{2}(\mathbb{Z})\right) \otimes \mathcal{A}
$$

This decomposition is respected by the operator $q \frac{d}{d q}$. Moreover $\mathcal{A}$ is a free polynomial algebra that is bi-graded with respect to weight and depth compatible with those of $\mathcal{Z}_{q}$. In particular it equals the graded dual to the universal enveloping algebra of a bi-graded Lie algebrd4.

This decomposition of algebras should be seen as an analogue of [11, Conjecture 1.1. b)] in our context. The conjecture above implies the weaker claim, that the algebra $\mathcal{Z}_{q}$ is isomorphic to a free polynomial algebra graded by the weight. It also implies that in Proposition 2.5 the equality holds.

Remark 2.7. In [17] Okounkov gives the following conjecture for the dimension of the weight-graded parts of $\mathcal{Z}_{q, 1}^{\circ}$.

$$
\sum_{k \geq 0} \operatorname{dim}_{\mathbb{Q}}\left(\operatorname{gr}_{k}^{\mathrm{W}} \mathcal{Z}_{q, 1}^{\circ}\right) x^{k} \stackrel{?}{=} \frac{1}{1-x^{2}-x^{3}-x^{4}-x^{5}+x^{8}+x^{9}+x^{10}+x^{11}+x^{12}} .
$$

We expect that the decomposition of Conjecture 2.6 induces also a decomposition for $\mathcal{Z}_{q, 1}^{\circ}$. Indeed, keeping the previous notation, this is compatible with the factorization

$$
\frac{1}{1-x^{2}-\ldots-x^{5}+x^{8}+\ldots+x^{12}}=\widetilde{\mathrm{M}}(x, 1) \frac{1}{1-\mathrm{D}(x) \mathrm{O}_{3}(x)+2 \mathrm{D}(x) \mathrm{S}(x)} .
$$

Our Conjecture 1.3 (i) for $\mathcal{Z}_{q}$ yields with $\mathrm{E}_{4}(x)=x^{4} /\left(1-x^{2}\right)$

$$
\frac{1}{1-x-x^{2}-x^{3}+x^{6}+\ldots+x^{9}}=\widetilde{\mathrm{M}}(x, 1) \frac{1}{1-\mathrm{D}(x) \mathrm{O}_{1}(x)+\mathrm{D}(x)\left(\mathrm{E}_{4}(x)+2 \mathrm{~S}(x)\right)} .
$$

Thus we may think of the Lie algebra behind $\mathcal{Z}_{q}$ compared to that behind $\mathcal{Z}_{q, 1}^{\circ}$ as having additional generators induced by the derivatives of a generator in weight 1 and having additional relations being counted by the number of Eisenstein series for $\mathrm{SL}_{2}(\mathbb{Z})$ and their derivatives.

\footnotetext{
${ }^{4}$ Some authors prefer to denote this as the symmetric algebra of a Lie algebra
} 


\section{Computational evidences for the conjectures}

In this section we want to describe how to implement the bi-brackets to obtain the numerical results, which were used to obtain Conjecture 1.3 in the introduction and further conjectures stated below. A similar method to perform such calculations has been communicated to us by Don Zagier.

Using (2.7) we define for a fixed $N \in \mathbb{N}$ an approximated version of bi-brackets by

$$
\left[\begin{array}{l}
s_{1}, \ldots, s_{l} \\
r_{1}, \ldots, r_{l}
\end{array}\right]_{N}:=\sum_{N \geq n_{1}>\cdots>n_{l}>0} \prod_{j=1}^{l}\left(\frac{n_{j}^{r_{j}}}{r_{j} !} \cdot \frac{q^{n_{j}} P_{s_{j}-1}\left(q^{n_{j}}\right)}{\left(s_{j}-1\right) ! \cdot\left(1-q^{n_{j}}\right)^{s_{j}}}\right) \in \mathbb{Q}[[q]] .
$$

Observe that $\left[\begin{array}{l}s_{1}, \ldots, s_{l} \\ r_{1}, \ldots, r_{l}\end{array}\right]_{N}=0$ for $N<l$. It is clear that at least the first $N$ coefficients of these approximated versions are identical to the bi-brackets, i.e.

$$
\left[\begin{array}{l}
s_{1}, \ldots, s_{l} \\
r_{1}, \ldots, r_{l}
\end{array}\right]_{N} \equiv\left[\begin{array}{l}
s_{1}, \ldots, s_{l} \\
r_{1}, \ldots, r_{l}
\end{array}\right] \quad \bmod q^{N+1}
$$

To calculate the first $N$ coefficients of the bi-brackets we use the following recursive formula for these approximated versions

Lemma 3.1. For all $s_{1}, \ldots, s_{l}, r_{1}, \ldots, r_{l}$ and $N \geq l$ we have

$$
\left[\begin{array}{l}
s_{1}, \ldots, s_{l} \\
r_{1}, \ldots, r_{l}
\end{array}\right]_{N}=\left[\begin{array}{l}
s_{1}, \ldots, s_{l} \\
r_{1}, \ldots, r_{l}
\end{array}\right]_{N-1}+\frac{N^{r_{1}}}{r_{1} !} \frac{q^{N} P_{s_{1}-1}\left(q^{N}\right)}{\left(s_{1}-1\right) ! \cdot\left(1-q^{N}\right)^{s_{1}}}\left[\begin{array}{l}
s_{2}, \ldots, s_{l} \\
r_{2}, \ldots, r_{l}
\end{array}\right]_{N-1},
$$

where we set $\left[\begin{array}{l}s_{2}, \ldots, s_{l} \\ r_{2}, \ldots, r_{l}\end{array}\right]_{N-1}=1$ for $l=1$.

Proof. This follows by splitting up the summation $N \geq n_{1}>\cdots>n_{l}>0$ into the parts where $N>n_{1}$ and $N=n_{1}$ to get the first and the second term respectively.

We implemented an algorithm based on Lemma 3.1 in parallel PARI/GP [23] and on a computer with 32 cores it takes several hours to obtain each of the following tables: 


\begin{tabular}{r|cccccccccccccc}
$k \backslash l$ & 1 & 2 & 3 & 4 & 5 & 6 & 7 & 8 & 9 & 10 & 11 & 12 & 13 & 14 \\
\hline 1 & 2 & 0 & 0 & 0 & 0 & 0 & 0 & 0 & 0 & 0 & 0 & 0 & 0 & 0 \\
2 & 3 & 4 & 0 & 0 & 0 & 0 & 0 & 0 & 0 & 0 & 0 & 0 & 0 & 0 \\
3 & 5 & 7 & 8 & 0 & 0 & 0 & 0 & 0 & 0 & 0 & 0 & 0 & 0 & 0 \\
4 & 7 & 12 & 14 & 15 & 0 & 0 & 0 & 0 & 0 & 0 & 0 & 0 & 0 & 0 \\
5 & 10 & 19 & 25 & 27 & 28 & 0 & 0 & 0 & 0 & 0 & 0 & 0 & 0 & 0 \\
6 & 13 & 30 & 41 & 48 & 50 & 51 & 0 & 0 & 0 & 0 & 0 & 0 & 0 & 0 \\
7 & 17 & 44 & 68 & 81 & 89 & 91 & 92 & 0 & 0 & 0 & 0 & 0 & 0 & 0 \\
8 & 21 & 65 & 106 & 138 & 153 & 162 & 164 & 165 & 0 & 0 & 0 & 0 & 0 & 0 \\
9 & 26 & 90 & 167 & 223 & 264 & 281 & 291 & 293 & 294 & 0 & 0 & 0 & 0 & 0 \\
10 & 31 & 126 & 249 & 366 & 439 & 490 & 509 & 520 & 522 & 523 & 0 & 0 & 0 & 0 \\
11 & 37 & 167 & 376 & 571 & 738 & 830 & 892 & 913 & 925 & 927 & 928 & 0 & 0 & 0 \\
12 & 43 & 222 & 537 & 905 & 1190 & 1418 & 1531 & 1605 & 1628 & 1641 & 1643 & 1644 & 0 & 0 \\
13 & 50 & 285 & 778 & 1364 & 1948 & 2344 & 2645 & 2781 & 2868 & 2893 & 2907 & 2909 & 2910 & 0 \\
14 & 57 & 368 & 1075 & 2090 & 3051 & 3923 & 4453 & 4840 & 5001 & 5102 & 5129 & 5144 & 5146 & 5147
\end{tabular}

Table 1: lower bounds fil $\ln _{k, l}^{\text {num }}\left(\mathcal{Z}_{q}\right)$ for $\operatorname{dim}_{\mathbb{Q}} \mathrm{Fil}_{k, l}^{\mathrm{W}, \mathrm{D}}\left(\mathcal{Z}_{q}\right)$ with depth $\leq 14$

\begin{tabular}{c|c|ccccccccccccccccccccc}
$l \backslash k$ & 1 & 2 & 3 & 4 & 5 & 6 & 7 & 8 & 9 & 10 & 11 & 12 & 13 & 14 & 15 & 16 & 17 & 18 & 19 & 20 & 21 \\
1 & 2 & 3 & 5 & 7 & 10 & 13 & 17 & 21 & 26 & 31 & 37 & 43 & 50 & 57 & 65 & 73 & 82 & 91 & 101 & 111 & 122 \\
2 & 0 & 4 & 7 & 12 & 19 & 30 & 44 & 65 & 90 & 126 & 167 & 222 & 285 & 368 & 460 & 577 & 706 & 866 & 1041 & 1254 & 1485 \\
3 & 0 & 0 & 8 & 14 & 25 & 41 & 68 & 106 & 167 & 249 & 376 & 537 & 778 & 1075 & 1503 & 2017 & 2737 & 3584 & 4739 & 6077 & 7859 \\
4 & 0 & 0 & 0 & 15 & 27 & 48 & 81 & 138 & 223 & 366 & 571 & 905 & 1364 & 2090 & 3053 & 4535 & 6440 & 9293 & $?$ & $?$ & $?$
\end{tabular}

Table 2: lower bounds $\operatorname{fil}_{k, l}^{\text {num }}\left(\mathcal{Z}_{q}\right)$ for $\operatorname{dim}_{\mathbb{Q}} \operatorname{Fil}_{k, l}^{\mathrm{W}, \mathrm{D}}\left(\mathcal{Z}_{q}\right)$ with depth $\leq 4$

In fact for these tables we calculated approximated bi-brackets with coefficients modulo some large prime and determined the dimension they span at least. Experimentally the choice of a sufficiently large prime does not alter the dimension. We have similar tables for various subspaces like the positive bi-brackets

$$
\mathcal{Z}_{q}^{+}=\left\langle\left[\begin{array}{l}
s_{1}, \ldots, s_{l} \\
r_{1}, \ldots, r_{l}
\end{array}\right] \in \mathcal{Z}_{q} \mid l \geq 0, s_{1}>r_{1}, \ldots, s_{l}>r_{l}\right\rangle_{\mathbb{Q}}
$$

or the space of 123-brackets given by

$$
\left\langle\left[s_{1}, \ldots, s_{l}\right] \mid l \geq 0, s_{1}, \ldots, s_{l} \in\{1,2,3\}\right\rangle_{\mathbb{Q}} \subset \mathcal{Z}_{q}^{\circ}
$$

and for sub-algebras like $\mathcal{Z}_{q}^{\circ}$ or $\mathcal{Z}_{q, 1}^{\circ}$. This lead us to the following conjectures

Conjecture 3.2. (B1) Every bi-bracket equals a linear combination of positive bi-brackets 
(B1*) More precisely, the space of positive bi-brackets $\mathcal{Z}_{q}^{+}$fulfills $\mathrm{Fil}_{w, l}^{\mathrm{W}, \mathrm{L}}\left(\mathcal{Z}_{q}^{+}\right)=\mathrm{Fil}_{w, l}^{\mathrm{W}, \mathrm{L}}\left(\mathcal{Z}_{q}\right)$.

(B2) Every bi-bracket equals a linear combination of brackets, i.e. $\mathcal{Z}_{q}^{\circ}=\mathcal{Z}_{q}$.

(B3) Every bracket equals a linear combination of 123-brackets.

Although, our experiments support conjectures (B1) and (B3), we were not able to prove the weaker claims that the positive bi-brackets respectively the 123-brackets generate sub-algebras of $\mathcal{Z}_{q}$. In [1] the conjecture (B2) was stated the first time and therein examples which complement those in [9] (Theorem 5.5) were given.

Theorem 3.3. For all weights $k \leq 7$ the coefficients on both sides of Conjecture 1.3 (i) coincide and in addition the Conjectures 3.2 (B1), (B2) and (B3) hold for these weights also.

We will give a proof of this theorem at the end of this section.

The Conjecture 1.3 is based on the assumption that the above lower bounds were the actual dimensions. In other words, for the quantities

$$
\operatorname{gr}_{k, l}^{\text {num }}=\operatorname{fil}_{k, l}^{\text {num }}\left(\mathcal{Z}_{q}\right)-\operatorname{fil}_{k, l-1}^{\text {num }}\left(\mathcal{Z}_{q}\right)-\operatorname{fil}_{k-1, l}^{\text {num }}\left(\mathcal{Z}_{q}\right)+\operatorname{fil}_{k-1, l-1}^{\text {num }}\left(\mathcal{Z}_{q}\right)
$$

we expect the equalities $\operatorname{gr}_{k, l}^{\text {num }}=\operatorname{dim}_{\mathbb{Q}} \operatorname{gr}_{k, l}^{\mathrm{W}, \mathrm{D}}\left(\mathcal{Z}_{q}\right)$. Now we check if the generating series of the weight- and depth-graded parts of $\mathcal{Z}_{q}$ can be of the shape implied by the conjectures. For example, if we assume that there is a decomposition $\mathcal{Z}_{q} \cong \widetilde{M}\left(\mathrm{SL}_{2}(\mathbb{Z})\right) \otimes$ $\mathcal{A}$, where the algebra $\mathcal{A}$ is a free polynomial algebra, then there must hold an equation of the form

$$
\sum_{k, l \geq 0} \operatorname{dim}_{\mathbb{Q}}\left(\operatorname{gr}_{k, l}\left(\mathcal{Z}_{q}\right)\right) x^{k} y^{l}=\widetilde{\mathrm{M}}(x, y) \cdot \prod_{k, l \geq 1} \frac{1}{\left(1-x^{k} y^{l}\right)^{g_{k, l}}},
$$

where the $g_{k, l}$ equal the number of generators of $\mathcal{A}$ in weight $k$ and depth $l$. Solving such an equation with with $\operatorname{gr}_{k, l}^{\text {num }}$ on the left-hand side, give us numerical $g_{k, l}^{\text {num }}$ and within the range of our experiments (See Table 3 on page 17) these are positive and satisfy a parity pattern. 


\begin{tabular}{|c|c|c|c|c|c|c|c|c|c|c|c|c|c|c|c|c|c|c|c|c|c|c|c|c|c|c|c|c|c|}
\hline $\operatorname{gr}_{k \backslash l}^{\mathrm{num}}$ & 1 & 2 & 3 & 4 & 5 & 6 & 7 & 8 & 9 & 10 & 11 & 12 & 13 & 14 & $g_{k \backslash l}^{\text {num }}$ & 1 & 2 & 3 & 4 & 5 & 6 & 7 & 8 & 9 & 10 & 11 & 12 & 13 & 14 \\
\hline 1 & $\mid 1$ & 0 & 0 & 0 & 0 & 0 & 0 & 0 & 0 & 0 & 0 & 0 & 0 & 0 & 1 & 1 & 0 & 0 & 0 & 0 & 0 & 0 & 0 & 0 & 0 & 0 & 0 & 0 & 0 \\
\hline 2 & $\mid 1$ & 1 & 0 & 0 & 0 & 0 & 0 & 0 & 0 & 0 & 0 & 0 & 0 & 0 & 2 & 0 & 0 & 0 & 0 & 0 & 0 & 0 & 0 & 0 & 0 & 0 & 0 & 0 & 0 \\
\hline 3 & 2 & 1 & 1 & 0 & 0 & 0 & 0 & 0 & 0 & 0 & 0 & 0 & 0 & 0 & 3 & 2 & 0 & 0 & 0 & 0 & 0 & 0 & 0 & 0 & 0 & 0 & 0 & 0 & 0 \\
\hline 4 & 12 & 3 & 1 & 1 & 0 & 0 & 0 & 0 & 0 & 0 & 0 & 0 & 0 & 0 & 4 & 0 & 1 & 0 & 0 & 0 & 0 & 0 & 0 & 0 & 0 & 0 & 0 & 0 & 0 \\
\hline 5 & $\mid 3$ & 4 & 4 & 1 & 1 & 0 & 0 & 0 & 0 & 0 & 0 & 0 & 0 & 0 & 5 & 3 & 0 & 1 & 0 & 0 & 0 & 0 & 0 & 0 & 0 & 0 & 0 & 0 & 0 \\
\hline 6 & $\mid 3$ & 8 & 5 & 5 & 1 & 1 & 0 & 0 & 0 & 0 & 0 & 0 & 0 & 0 & 6 & 0 & 2 & 0 & 1 & 0 & 0 & 0 & 0 & 0 & 0 & 0 & 0 & 0 & 0 \\
\hline 7 & | 4 & 10 & 13 & 6 & 6 & 1 & 1 & 0 & 0 & 0 & 0 & 0 & 0 & 0 & 7 & 4 & 0 & 3 & 0 & 1 & 0 & 0 & 0 & 0 & 0 & 0 & 0 & 0 & 0 \\
\hline 8 & $\mid 4$ & 17 & 17 & 19 & 7 & 7 & 1 & 1 & 0 & 0 & 0 & 0 & 0 & 0 & 8 & 0 & 7 & 0 & 3 & 0 & 1 & 0 & 0 & 0 & 0 & 0 & 0 & 0 & 0 \\
\hline 9 & 5 & 20 & 36 & 24 & 26 & 8 & 8 & 1 & 1 & 0 & 0 & 0 & 0 & 0 & 9 & 5 & 0 & 8 & 0 & 4 & 0 & 1 & 0 & 0 & 0 & 0 & 0 & 0 & 0 \\
\hline 10 & $\mid 5$ & 31 & 46 & 61 & 32 & 34 & 9 & 9 & 1 & 1 & 0 & 0 & 0 & 0 & 10 & 0 & 12 & 0 & 11 & 0 & 4 & 0 & 1 & 0 & 0 & 0 & 0 & 0 & 0 \\
\hline 11 & | 6 & 35 & 86 & 78 & 94 & 41 & 43 & 10 & 10 & 1 & 1 & 0 & 0 & 0 & 11 & 6 & 0 & 22 & 0 & 14 & 0 & 5 & 0 & 1 & 0 & 0 & 0 & 0 & 0 \\
\hline 12 & | 6 & 49 & 106 & 173 & 118 & 136 & 51 & 53 & 11 & 11 & 1 & 1 & 0 & 0 & 12 & 0 & 20 & 0 & 31 & 0 & 17 & 0 & 5 & 0 & 1 & 0 & 0 & 0 & 0 \\
\hline 13 & | 7 & 56 & 178 & 218 & 299 & 168 & 188 & 62 & 64 & 12 & 12 & 1 & 1 & 0 & 13 & 7 & 0 & 47 & 0 & 44 & 0 & 21 & 0 & 6 & 0 & 1 & 0 & 0 & 0 \\
\hline 14 & 7 & 76 & 214 & 429 & 377 & 476 & 229 & 251 & 74 & 76 & 13 & 13 & 1 & 1 & 14 & 0 & 31 & 0 & 81 & 0 & 58 & 0 & 25 & 0 & 6 & 0 & 1 & 0 & 0 \\
\hline
\end{tabular}

Table 3: Evidence for $\mathcal{A}$ being a free polynomial algebra

If we assume that there is a decomposition $\mathcal{Z}_{q} \cong \widetilde{M}\left(\mathrm{SL}_{2}(\mathbb{Z})\right) \otimes \mathcal{A}$, where the algebra $\mathcal{A}$ is the graded dual to the universal enveloping algebra of a bi-graded Lie algebra, then there must hold an equation of the form

$$
\sum_{k, l \geq 0} \operatorname{dim}_{\mathbb{Q}}\left(\operatorname{gr}_{k, l}^{\mathrm{W}, \mathrm{D}}\left(\mathcal{Z}_{q}\right)\right) x^{k} y^{l}=\tilde{\mathrm{M}}(x, y) \cdot \frac{1}{1-\sum_{k, l \geq 1} b_{k, l} x^{k} y^{l}}
$$

with $b_{k, l} \in \mathbb{Z}$. Solving such an equation with with $\operatorname{gr}_{k, l}^{\text {num }}\left(\mathcal{Z}_{q}\right)$ on the left-hand side, give us numerical $b_{k, l}^{\text {num }}$ and within the range of our experiments (See Table 4 on page 17) these are as expected in Conjecture 1.3 (ii)

\begin{tabular}{c|c|c|c|c|c|c|c|c|c|c|c|c|c|c}
$l$ & 1 & 2 & 3 & 4 & 5 & 6 & 7 & 8 & 9 & 10 & 11 & 12 & 13 & 14 \\
\hline$k \leq$ & 63 & 31 & 21 & 19 & 15 & 14 & 14 & 14 & 14 & 14 & 14 & 14 & 14 & 14
\end{tabular}

Table 4: Evidence for $\mathcal{A}$ being a symmetric algebra of a Lie algebra

Whereas it is known that the numbers from Zagier's conjecture give upper bound for the dimensions in question, the knowledge about the Broadhurst-Kreimer conjecture is very little. The only known results are the following:

Theorem 3.4 (Euler, Ihara-Kaneko-Zagier, Goncharov, Ihara-Ochiai). For $1 \leq l \leq 3$ the numbers $g_{k, l}$ of generators for $\mathcal{Z}$ of weight $k$ and depth $l$ are not bigger than implied by the Broadhurst-Kreimer conjecture.

The proof of this result for $l=1$ is a trivial consequence of Euler's formula for even zeta values. For $l=2,3$ one can bound the number of generators by the dimension of the so called double shuffle spaces, see e.g. [12], [11], [15] . 
We now want to use a similar technique to obtain upper bounds of the number of algebra generators for bi-brackets.

For the generating function of the bi-brackets we write

$$
\left|\begin{array}{c}
X_{1}, \ldots, X_{l} \\
Y_{1}, \ldots, Y_{l}
\end{array}\right|:=\sum_{\substack{s_{1}, \ldots, s_{l}>0 \\
r_{1}, \ldots, r_{l}>0}}\left[\begin{array}{c}
s_{1}, \ldots, s_{l} \\
r_{1}-1, \ldots, r_{l}-1
\end{array}\right] X_{1}^{s_{1}-1} \ldots X_{l}^{s_{l}-1} \cdot Y_{1}^{r_{1}-1} \ldots Y_{l}^{r_{l}-1}
$$

As shown in [1] this satisfies the partition relation

$$
\left|\begin{array}{c}
X_{1}, \ldots, X_{l} \\
Y_{1}, \ldots, Y_{l}
\end{array}\right|=\left.\left|\begin{array}{c}
X_{1}, \ldots, X_{l} \\
Y_{1}, \ldots, Y_{l}
\end{array}\right|\right|_{P}
$$

with $\left.f\left(X_{1}, \ldots, X_{l}, Y_{1}, \ldots, Y_{l}\right)\right|_{P}=f\left(Y_{1}+\cdots+Y_{l}, \ldots, Y_{1}+Y_{2}, Y_{1}, X_{l}, X_{l}-X_{l-1}, \ldots, X_{2}-X_{1}\right)$. Up to terms of depth less than $l$ their product is given by

$$
\left|\begin{array}{c}
X_{1}, \ldots, X_{j} \\
Y_{1}, \ldots, Y_{j}
\end{array}\right| \cdot\left|\begin{array}{c}
X_{j+1}, \ldots, X_{l} \\
Y_{j+1}, \ldots, Y_{l}
\end{array}\right|=\left.\left|\begin{array}{c}
X_{1}, \ldots, X_{l} \\
Y_{1}, \ldots, Y_{l}
\end{array}\right|\right|_{\mathrm{Sh}_{j, l}}+\ldots
$$

where, if $\Sigma_{j, l} \subset \Sigma_{n}$ denotes the shuffles of ordered sets with $j$ and $l-j$ elements, we have

$$
\left.f\left(X_{1}, \ldots, X_{l}, Y_{1}, \ldots, Y_{l}\right)\right|_{\mathrm{Sh}_{j, l}}=\sum_{\sigma \in \Sigma_{j, l}} f\left(X_{\sigma^{-1}(1)}, \ldots, X_{\sigma^{-1}(l)}, Y_{\sigma^{-1}(1)}, \ldots, Y_{\sigma^{-1}(l)}\right)
$$

Hence we get modulo products and lower depth bi-brackets

$$
\left|\begin{array}{c}
X_{1}, \ldots, X_{l} \\
Y_{1}, \ldots, Y_{l}
\end{array}\right| \equiv \sum_{\alpha} \alpha F_{\alpha}\left(X_{1}, \ldots, X_{l}, Y_{1}, \ldots, Y_{l}\right)
$$

where $\alpha$ runs through a vector space basis of the depth $l$ algebra generators of $\mathcal{Z}_{q}$ and $F_{\alpha}$ is a polynomial in the partition shuffle space, which is defined as follows.

Definition 3.5. Define for $l, k \geq 0$ the partition shuffle space by

$$
\mathbb{P} \mathbb{S}(k-l, l)=\left\{f \in \mathbb{Q}\left[x_{1}, . ., x_{l}, y_{1}, . ., y_{l}\right]|\operatorname{deg} f=k-l, f|_{P}-f=\left.f\right|_{\mathrm{Sh}_{j}}=0 \forall j\right\} .
$$

Using the same argument as in [12] the above discussion leads to the following upper bounds.

Corollary 3.6. The number $g_{k, l}$ of generators of weight $k$ and depth $l$ for the $\mathbb{Q}$-algebra 
$\mathcal{Z}_{q}$ is bounded by

$$
g_{k, l} \leq \operatorname{dim}_{\mathbb{Q}} \mathbb{P S}(k-l, l)
$$

The bounds obtained via the partition shuffle spaces for the number of generators in depth 1 and even weights are not optimal, as it is well-known that the ring of quasimodular forms is generated in weight 2,4 and 6 . We view this as the analogue to the fact that Euler's relation for even zeta values is not seen by the depth 1 double shuffle spaces as defined in [12].

\begin{tabular}{c|c|c|c|c|c|c|c|c|c|c|c|c|c|c|c|c|c|}
$p_{l \backslash k}$ & 1 & 2 & 3 & 4 & 5 & 6 & 7 & 8 & 9 & 10 & 11 & 12 & 13 & 14 & 15 & 16 & 17 \\
\hline 1 & 1 & 1 & 2 & 2 & 3 & 3 & 4 & 4 & 5 & 5 & 6 & 6 & 7 & 7 & 8 & 8 & 9 \\
\hline 2 & - & 0 & 0 & 1 & 0 & 2 & 0 & 8 & 0 & 14 & 0 & 23 & 0 & 38 & 0 & 58 & 0 \\
\hline 3 & - & - & 0 & 0 & 1 & 0 & 3 & 0 & 9 & 0 & 27 & 0 & 62 & 0 & 125 & 0 & 238 \\
\hline 4 & - & - & - & 0 & 0 & 1 & 0 & 3 & 0 & 12 & 0 & 37 & $?$ & $?$ & $?$ & $?$ & $?$ \\
\hline 5 & - & - & - & - & 0 & 0 & 1 & 0 & 4 & 0 & 15 & $?$ & $?$ & $?$ & $?$ & $?$ & $?$ \\
\hline 6 & - & - & - & - & - & 0 & 0 & 1 & $?$ & $?$ & $?$ & $?$ & $?$ & $?$ & $?$ & $?$ & $?$ \\
\hline
\end{tabular}

Table 5: $\quad p_{k, l}=\operatorname{dim}_{\mathbb{Q}} \mathbb{P} \mathbb{S}(k-l, l)$

Proof of Theorem 3.3 Using the structure of the ring of quasi-modular forms and the data of Table 5 we get the coefficient-wise upper bounds

$$
\begin{gathered}
\sum_{k \geq 0} \operatorname{dim}_{\mathbb{Q}} \operatorname{Fil}_{k}^{\mathrm{W}}\left(\mathcal{Z}_{q}\right) x^{k} \leq \frac{1}{1-x} \frac{1}{\left(1-x^{2}\right)\left(1-x^{4}\right)\left(1-x^{6}\right)} \frac{x}{\left(1-x^{2}\right)^{2}} \cdot \prod_{k, l \geq 2} \frac{1}{\left(1-x^{k}\right)^{p_{k, l}}} \\
\leq 1+2 x+4 x^{2}+8 x^{3}+15 x^{4}+28 x^{5}+51 x^{6}+92 x^{7}+166 x^{8}+\ldots
\end{gathered}
$$

In addition, since "123-brackets" $\subseteq \mathcal{Z}_{q}^{\circ} \subseteq \mathcal{Z}_{q}$, we get by the data of our tables

$$
\begin{aligned}
& 1+2 x+4 x^{2}+8 x^{3}+15 x^{4}+28 x^{5}+51 x^{6}+92 x^{7}+165 x^{8}+\ldots \\
& \leq \sum_{k \geq 0} \operatorname{dim}_{\mathbb{Q}} \mathrm{Fil}_{k}^{\mathrm{W}}\left(" 123 \text {-brackets") } x^{k} \leq \sum_{k \geq 0} \operatorname{dim}_{\mathbb{Q}} \mathrm{Fil}_{k}^{\mathrm{W}}\left(\mathcal{Z}_{q}\right) x^{k} .\right.
\end{aligned}
$$

The claim of the theorem follows as the lower and upper bounds coincide for $k \leq 7$.

Remark 3.7. In contrast to the multiple zeta values we expect that the upper bounds for the number of generators obtained by the partition shuffle spaces are not optimal for all $l \geq 2$, i.e. we don't expect equality in Corollary 3.6. We think that this reflects the existence of cusp forms as distinguished elements in depth 2, whereas even zeta values just live in depth 1. By work of Ecalle we know that there is a Lie algebra structure on the partition shuffle spaces, see e.g. [8] or [19]. In forthcoming work we will study a sub 
Lie algebra which conjecturally has the algebra $\mathcal{A}$ as its symmetric algebra, which might give another explanation of this effect. Another optimistic hope is that a coproduct structure on $\mathcal{Z}_{q}$, which allows to mimic Brown's proof in order to obtain conjecture (B3), exists.

\section{References}

[1] H. Bachmann: The algebra of bi-brackets and regularized multiple Eisenstein series, arXiv:1504.08138 [math.NT].

[2] H. Bachmann: Multiple Eisenstein series and q-analogues of multiple zeta values, to appear in "Periods in Quantum Field Theory and Arithmetic", arXiv:1704.06930 [math.NT].

[3] H. Bachmann: Double shuffle relations for q-analogues of multiple zeta values, their derivatives and the connection to multiple Eisenstein series, RIMS Kôyûroku No. 2015 (2017), 22-43.

[4] H. Bachmann, U. Kühn: The algebra of generating functions for multiple divisor sums and applications to multiple zeta values, Ramanujan J. 40 (2016), 605-648.

[5] D. M. Bradley: Multiple q-zeta values, J. Algebra, 283 (2005), 752-798.

[6] D. Broadhurst, D. Kreimer: Association of multiple zeta values with positive knots via Feynman diagrams up to 9 loops, Phys. Lett. B 393 (1997), 403-412.

[7] F. Brown: Mixed Tate motives over $\mathbb{Z}$, Ann. of Math. (2) 175 (2012), 949-976.

[8] J. Ecalle: The flexion structure and dimorphy: flexion units, singulators, generators, and the enumeration of multizeta irreducibles, Asymptotics in dynamics, geometry and PDEs, generalized Borel summation. Vol. II (2011), 27-211.

[9] K. Ebrahimi-Fard, D. Manchon, J. Singer, Duality and (q-)multiple zeta values, Adv. Math. 298 (2016), 254-285.

[10] D. Foata: Eulerian polynomials: from Euler's Time to the Present, The legacy of Alladi Ramakrishnan in the mathematical sciences, Springer, New York (2010), 253-273.

[11] A. B. Goncharov, Multiple $\zeta$-values, Galois groups and geometry of modular varieties, Progr. Math. 201 (2001), 361-392. 
[12] K. Ihara, M. Kaneko, D. Zagier: Derivation and double shuffle relations for multiple zeta values, Compositio Math. 142 (2006), 307-338.

[13] M.E. Hoffman: The Algebra of Multiple Harmonic Series, J. Algebra 194 (1997), 477-495.

[14] M.E. Hoffman, K. Ihara: Quasi-shuffle products revisited, J. Algebra 481 (2017), 293-326.

[15] K. Ihara, H. Ochiai: Symmetry on linear relations for multiple zeta values, Nagoya Math. J. 189 (2008), 49-62.

[16] M. Kaneko, D. Zagier: A generalized Jacobi theta function and quasimodular forms, The moduli space of curves, Progr. Math. 129 (1995), 165-172.

[17] A. Okounkov: Hilbert schemes and multiple q-zeta values, Funct. Anal. Appl. 48 (2014), 138-144.

[18] K. Schlesinger: Some remarks on q-deformed multiple polylogarithms, arXiv:math/0111022 [math.QA].

[19] L. Schneps: ARI, GARI, Zig and Zag: An introduction to Ecalle's theory of multiple zeta values, arXiv:1507.01534 [math.NT].

[20] J. Singer: On q-analogues of multiple zeta values, Funct. Approx. Comment. Math. 53 (2015), 135-165.

[21] Y. Takeyama, The algebra of a q-analogue of multiple harmonic series. SIGMA 9 Paper 061 (2013), 1-15.

[22] Y. Ohno, J. Okuda, W. Zudilin: Cyclic $q-M Z S V$ sum. J. Number Theory 132 (2012), 144-155.

[23] The PARI Group, PARI/GP version 2.10.0, Univ. Bordeaux, 2017, http://pari.math.u-bordeaux.fr/.

[24] Y. Pupyrev: On the linear and algebraic independence of q-zeta values, (Russian. Russian summary) Mat. Zametki 78 (2005), no. 4, 608-613; translation in Math. Notes 78 (2005), no. 3-4, 563-568.

[25] D. Zagier: Values of zeta functions and their applications, First European Congress of Mathematics, Volume II, Progress in Math. 120, Birkhäser-Verlag, Basel (1994), 497-512. 
[26] J. Zhao: Multiple q-zeta functions and multiple q-polylogarithms, Ramanujan J. $14(2)$ (2007), 189-221.

[27] J. Zhao: Uniform approach to double shuffle and duality relations of various q-analogs of multiple zeta values via Rota-Baxter algebras, arXiv:1412.8044 [math.NT].

[28] W. Zudilin: Diophantine problems for q-zeta values, (Russian) Mat. Zametki 72 (2002), no. 6, 936-940; translation in Math. Notes 72 (2002), 858-862.

[29] W. Zudilin: Algebraic relations for multiple zeta values, Russian Math. Surveys 58(1) (2003), 1-29.

[30] W. Zudilin: Multiple q-zeta brackets, Mathematics 3:1, special issue Mathematical physics (2015), 119-130.

Henrik Bachmann, Nagoya University.

henrik.bachmann@math.nagoya-u.ac.jp

Ulf Kühn, Universität Hamburg.

kuehn@math.uni-hamburg.de 